\title{
Plasticity moves upstream
}

Neuronal activity can increase or decrease the strength of synapses between two neurons through long-term potentiation (LTP) and long-term depression (LTD), respectively. Now, Poo and colleagues show that, in vivo, LTP and LTD can spread to the upstream synapse.

In vitro studies had shown that LTP and LTD induced in a particular synapse could modify adjacent synapses, but it was not known whether this also occurs in vivo. Poo and colleagues investigated this question in the visual system of the tadpole by inducing LTP and LTD at output synapses of retinal ganglion cells (RGCs) in the optic tectum and measuring the

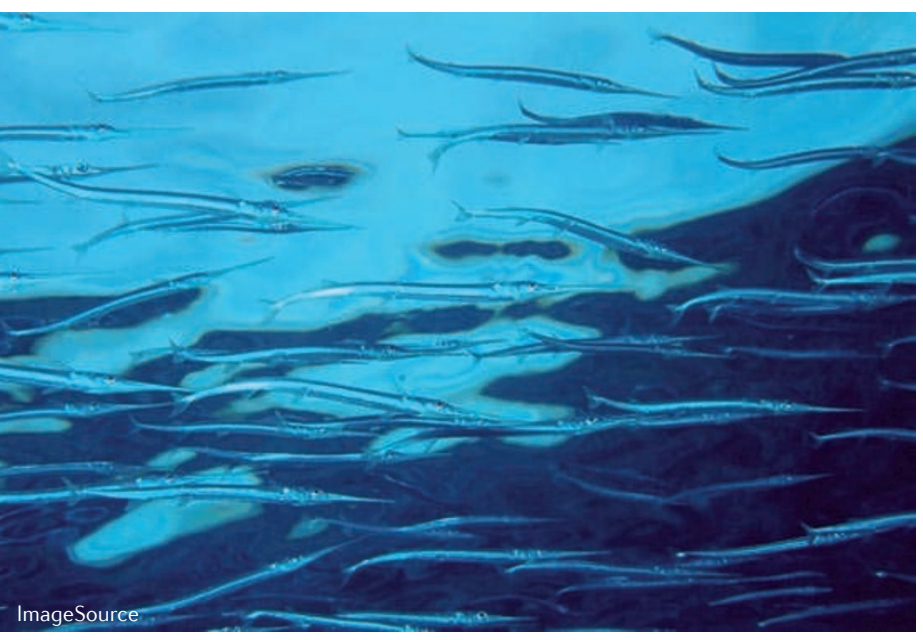

strength of synapses between bipolar cells (BCs) and RGCs.

The authors used theta burst stimulation and low-frequency stimulation of RGC axons to induce LTP and LTD, respectively, at retinotectal synapses. They then investigated whether this affected light-evoked excitatory compound synaptic currents (eCSCs) at BC-RGC synapses. Retinotectal LTP and LTD induction respectively enhanced and reduced eCSCs for $\sim 20 \mathrm{~min}$ (or up to $1 \mathrm{~h}$ when spaced stimulation protocols were used). Only BC synapses with RGCs that had undergone LTP or LTD showed potentiation or depression, indicating that plasticity 'spreads' retrogradely within the RGC but not to adjacent RGCs. Interestingly, pre-incubating the tadpole with a microtubule-disrupting agent prevented the eCSC changes, suggesting that axonal transport along microtubules might mediate the spread of LTP and LTD.

Aiming to identify the retrograde signals, the authors found that brain-derived neurotrophic factor signalling through TrkB was required not only for LTP at retinotectal synapses but also for its retrograde spread: downregulating or blocking presynaptic or postsynaptic TrkB receptors reduced retinotectal LTP, and blocking presynaptic TrkB activity in RGCs prevented the
LTP-induced potentiation of BC-RGC synapses.

By contrast, LTD induction at retinotectal synapses and its retrograde spread required nitric oxide (NO) synthesis and signalling. Reducing the level or synthesis of NO in the tectum neuron prevented retinotectal LTD induction, and extracellular loading (by intraventricular infusion) of an NO scavenger prevented the spread of LTD to BC-RGC synapses. The retrograde LTD spread also required the activity of a downstream component of the NO signalling pathway, protein kinase $\mathrm{G}$, in the RGC, suggesting that NO from the tectal neuron diffuses to the presynaptic RGC, where it might activate protein kinase $\mathrm{G}$ and so eventually induce depression at BC-RGC synapses.

The spread of synaptic modifications along a neuron away from the initial site of induction might permit the coordination of synaptic changes in neural circuits during developmental refinement or after learning in adulthood. Whether retrograde, 'secondary' plasticity can spread further up or also down a circuit remains to be investigated.

Leonie Welberg

ORIGINAL RESEARCH PAPER Du, J.-l. et al. Long-range retrograde spread of LTP and LTD from optic tectum to retina. Proc. Natl Acad. Sci. USA 106, 18890-18896 (2009) 